\section{PREDICCIONES Y PERCEPCIÓN DE RIESGO SOCIAL. LOS PRONÓSTICOS FALLIDOS SOBRE LA CRISIS DE LAS PENSIONES PÚBLICAS ESPAÑOLAS}

\author{
Pablo Francescutti \\ Universidad Rey Juan Carlos \\ luispablo.francescutti@urjc.es \\ ORCID iD: http://orcid.org/0000-0002-5369-2835
}

\section{PREDICTIONS AND SOCIAL RISK PERCEPTION. THE FLAWED FORECASTS ABOUT THE SPANISH PUBLIC PENSIONS CRISIS}

Cómo citar este artículo/Citation: Francescutti, P. (2017). Predicciones y percepción de riesgo social. Los pronósticos fallidos sobre la crisis de las pensiones públicas españolas. Arbor, 193 (784): a383. doi: http://dx.doi.org/10.3989/ arbor.2017.784n2006

Recibido: 17 junio 2014. Aceptado: 9 mayo 2016.

RESUMEN: El debate en España sobre la viabilidad del Estado de Bienestar tiene entre sus principales ingredientes a las recurrentes predicciones catastrofistas sobre las pensiones públicas. En este artículo se analiza una serie de vaticinios formulados a mediados de los años ‘90. El cumplimiento de los plazos previstos en sus pronósticos permite cotejar retrospectivamente su grado de cumplimiento y la dimensión real del riesgo imputado a esa forma de seguro colectivo con la evolución histórica de las cuentas del sistema de pensiones. El análisis de su metodología, sus objetivos, sus recomendaciones y los intereses de sus patrocinadores ilumina su contribución a la percepción del riesgo de colapso de la Seguridad Social, sus posibles efectos reflexivos, así como la incidencia de las predicciones en la definición de los riesgos sociales.

PALABRAS CLAVE: predicciones; riesgo; pensiones; reflexividad; estado de bienestar.
Copyright: (C 2017 CSIC. Este es un artículo de acceso abierto distribuido bajo los términos de la licencia Creative Commons Attribution (CC BY) España 3.0.
ABSTRACT: The debate in Spain about the Welfare State sustainability is fuelled by recurrent catastrophic predictions about the future of public pensions. In this paper a set of such predictions made in the mid-1990s is analyzed. In retrospect, their accuracy is evaluated in relation to the historical evolution of the Spanish public finance. In the same way, the real dimension of pensions risks is assessed. Moreover, their methodology, aims and the recommendations made by their authors are discussed. The results shed light on the influence of these predictions upon risk perception of financial collapse of Social Security, on their reflexive effects as well as on their impact on the process of definition of risks.

KEYWORDS: predictions; risks; public pensions; reflexivity; Welfare State. 


\section{CONSIDERACIONES PREVIAS: RIESGO Y PREDICCIÓN}

A principios de los años '90, un nuevo riesgo social saltó a la palestra: el de la crisis de las pensiones públicas. Lo avalaba una serie de predicciones sobre el impacto del envejecimiento de la población elaboradas por el Banco Mundial (Palacios, 1994) y la Organización para la Cooperación y el Desarrollo Económicos (OCDE) (Van den Noord y Herd, 1993; Van den Noord y Herd, 1994). Enfrentadas a este "problema", todas coincidían en el pronóstico -el aumento de las clases pasivas gravará de modo insostenible el estado de bienestar y la economía- y en la prescripción -restricciones en la sanidad pública, aumento de la edad de retiro, disminución de la cuantía y cobertura de la jubilación estatal e impulso de las pensiones privadas.

La irrupción de dicho riesgo había sido abonada por la corriente neomalthusiana que desde los años '80 venía pregonando las consecuencias catastróficas del envejecimiento (Preston, 1984; Callahan, 1987). Su conexión con la política social resultaba funcional al cuestionamiento del estado providente, en la medida en que, para el neoliberalismo, "Ios seguros sociales de todo tipo, y demás dispositivos que han eliminado el acicate propinado por la necesidad de velar por uno mismo en una competencia abierta, deben ser desmantelados y reemplazados por acuerdos privados" (O’Mailly, 1996, p. 196). A la socialización de los riesgos que afrontaban los trabajadores (Ewald, 1986), aquella doctrina opone la confianza plena en la mano invisible del mercado (Kennedy, 2009) al tiempo que en el plano individual prescribe "una subjetividad afín con las racionalidades políticas neoliberales, así como con las nuevas tecnologías del riesgo" (Sepúlveda Galeas, 2011, p. 111). Se reafirma así el principio de responsabilidad con el propósito de convertir al individuo en gestor de sus riesgos. Libre de la tutela estatal, el sujeto prudente, responsable y racional se ve obligado a la toma de decisiones que le garanticen seguridad mediante el concurso de expertos cuyos servicios contratará si quiere evitar el desamparo en el que amenaza dejarlo un estado de bienestar abocado a la ruina. Para empoderarlo se implementan "dispositivos pedagógicos a través de los cuales se dotará a los individuos de un equipamiento semiótico-material para que estos puedan gestionar de forma eficaz los riesgos. La pedagogía del riesgo se lleva a cabo mediante un package formativo que interviene a nivel cognitivo, conductual y actitudinal a través de la entrega de información basada en evidencias científicas disponibles" (Sepúlveda Galeas, 2011, pp. 120-121). Entre estas se incluyen las predicciones sobre pensiones, las cuales, con la fuerza de las cifras demográficas y una mezcla de pesimismo respecto al futuro de las finanzas públicas y de optimismo sobre la sagacidad inversora del individuo, buscan enseñarle a gestionar las incertidumbres ligadas a la vejez. En la práctica ha ocurrido que, a despecho de la "democratización de los riesgos" postulada por Beck (1998, p. 46 y ss.), los riesgos se han ido distribuyendo de forma cada vez más regresiva, con el resultado de que las clases populares se tornan más vulnerables al albur económico mientras las élites viven protegidas por su riqueza y sus seguros privados.

Que el riesgo de quiebra de las pensiones hiciera cundir la alarma pese a que ningún país miembro de la OCDE había dejado hasta ese momento de pagar sus jubilaciones dio pie a que se tachara a las predicciones de "demografía apocalíptica" por pivotar "en la visión de que un número creciente de personas mayores llevará la sociedad a la quiebra, debido a sus incesantes demandas al sistema de salud y a las pensiones públicas" (Gee y Gutman, 2000, p. 6). Se les acusó asimismo de propagar la "retórica de la bancarrota" (Jerit y Barabas, 2006) con designios persuasivos: "en Gran Bretaña y en toda Europa la pensión estatal es una institución popular extremadamente popular. Suprimirla o reducirla suscita una oposición masiva. Solo desmoralizando a la población con la creencia en su insostenibilidad demográfica se abre espacio a la creación de un mercado masivo de pensiones" (Vincent, 2003, p. 86). Robert Castel denunció el uso de la "ideología generalizada e indiferenciada del riesgo" como ariete contra la protección estatal, y en particular la inflación de los riesgos, práctica que "abre un mercado prácticamente infinito al comercio de los seguros" (Castel, 2004, p. 83).

La polémica quedó planteada. Unos sostenían que la posibilidad de una insolvencia de la Seguridad Social era una realidad creada por la mayor esperanza de vida y el creciente número de titulados a recibir pensiones; otros arguían que era un debate artificioso generado para desmantelar las redes de protección social. Dado que la discusión giraba en torno a predicciones que no habían pasado la prueba de los hechos, resultaba difícil dirimir quién llevaba razón. En última instancia, la dificultad trascendía la controversia, pues radicaba en la dimensión virtual del riesgo, al que Niklas Luhmann (2006) define como la posibilidad de sufrir daños debido a decisiones tomadas en el presente (p. 62), la Royal Society (1992) como "la posibilidad, en términos cuantitativos, de la ocurrencia de un peligro determinado" (p. 4), y Anthony Giddens como "peligros que son activamente valorados en relación a posibilidades futuras" (BBC Reith Lectures 1999). 
La cualidad hipotética, virtual e inmaterial del riesgo arroja un reto formidable a las estrategias encaminadas a delimitarlo, tasarlo y evaluarlo, pues es de su medición y de la comprensión rigurosa de las cadenas de consecuencias y reacciones de donde se derivará el nivel de gravedad expresado en términos de consecuencias probables (Lupton, 1999). Mas ocurre que los riesgos versan sobre daños futuros, y es sabido desde Aristóteles (Candel Sanmartín, 1988, p. 52 y ss.) que de los enunciados sobre el porvenir no se puede predicar la verdad ni la falsedad. $Y$ a despecho de la indecibilidad que afecta a los asertos sobre riesgos, los analistas objetivistas no dejan de atribuir a sus predicciones y cálculos probabilísticos el estatuto de hechos ineluctables y verdades científicas (Bradbury, 1989). De lo expuesto se deduce el rol capital en la definición del riesgo social de las predicciones demográficas y económicas. Las primeras consisten en "una proyección basada en hipótesis muy probables sobre el comportamiento futuro de los fenómenos demográficos" (Diccionario demográfico multilingüe, s. v. Predicción demográfica) y en la premisa de que las variables de mortalidad, fertilidad y migraciones se mantendrán constantes a lo largo de un periodo. Asumir que el futuro será igual al pasado reciente no reduce su grado de acierto en el corto plazo -las probabilidades de evolución continua de una tendencia son mayores que las de su ruptura brusca- pero sí lo debilita en el mediano y largo plazo -por excluir mutaciones históricas, contingencias y estructuras emergentes, entre otras eventualidades-. De tal guisa actúa la falacia malthusiana al "asumir que un único cambio demográfico causará estragos en la sociedad basándose solamente en dicho cambio y sin tener en cuenta otros cambios que podrían darse" (Markert, 2005, p. 565).

Todas esas características se tornan mucho más acusadas en la predicción económica, pues esta, en razón de la complejidad del sistema económico, maneja un número elevado de variables, lo que conspira contra su eficacia. Otro tanto ocurre con su dependencia de las series históricas, ya que "extrapolar promedios pasados al futuro es un ejercicio arriesgado. Toda variable económica tiene puntos de inflexión y el elemento más importante de una predicción a largo plazo es determinar cuándo se producirán las inflexiones" (Zubiri Oria, 2003, p. 53). Por esa razón, "quienes pronostican valiéndose de modelos informáticos no se desempeñan mejor que quienes se basan solo en su juicio subjetivo, y quienes utilizan vastos modelos con más de mil ecuaciones no lo hacen mejor que quienes aplican modelos simples con pocas ecuaciones" (Sherden, 1998, p. 66).
Las limitaciones intrínsecas de la predicción, sumadas a su incapacidad para captar la complejidad de los riesgos no mensurables (Wynne, 1992, p. 296), no le han impedido satisfacer la demanda de escenarios futuros generada por la planificación, la contabilidad nacional y los procesos decisorios. Los vaticinios demográficos y económicos, al proporcionar elementos que justifican decisiones racionales en condiciones de incertidumbre en base a la confianza que inspira la descripción objetiva de variables operativas en el presente y la racionalidad de su procedimiento, son de uso corriente en el diseño y el debate sobre políticas económicas, educativas y sociales.

Para la sociología la relevancia de las predicciones no pasa tanto por lo adecuado de su metodología como por su potencia creadora de hechos sociales. Aparte de reducir la apertura del mañana a unas pocas opciones probables y manejables (Luhmann, 1992, p. 172 y ss.), pueden influir en el devenir de lo vaticinado. A esta facultad se referían las profecías sociales teorizadas por Merton (1948): descripciones de estados futuros que, al tornarse conocimiento social, propician su advenimiento (profecías auto-cumplidas) - los abortan (profecías suicidas). Para que una predicción surta tales efectos debe cobrar carácter público, pues difícilmente un pronóstico secreto desencadenará reacciones colectivas dirigidas a alcanzar o frustrar el escenario anunciado.

La importancia de esa propiedad de cara al riesgo se hace patente toda vez que "los debates sobre definiciones de riesgo y sus consecuencias para la sociedad tienen lugar esencialmente al nivel de los discursos públicos o parcialmente públicos" (Lau, citado en Beck, 1996, p. 36). Al difundirse, las predicciones pueden modular las percepciones incitando o inhibiendo acciones en los individuos concernidos. Reflexividad predictiva y riesgo guardan una relación dialéctica: la predicción que enfatiza la gravedad de un riesgo puede desatar reacciones que prueben a posteriori que tenía fundamento o, al contrario, su irrelevancia. Quienes las formulan suelen conocer su valor como acicate de la reflexividad social, y en ocasiones las diseñan en función de las reacciones que buscan conseguir con ellas $^{1}$. Resulta entonces pertinente preguntarse si los autores de los pronósticos sobre pensiones tuvieron en cuenta sus efectos reflexivos con miras a la consecución de sus fines; vale decir, si exageraron el riesgo financiero para sacar adelante su agenda política.

Lo expuesto amerita el interés de las predicciones sobre pensiones elaboradas en España en la estela del Banco Mundial y la OCDE. Por añadidura, su difusión 
en un contexto de superávits del sistema contributivo (Iglesias Fernández, 2010, p. 52) y de debate sobre las pensiones y el estado de bienestar (Zubiri Oria, 2003; Martínez Noval y Sáenz de Jubera Álvarez, 2009; Esteve Mora y Muñoz de Bustillo Llorente, 2004), la posibilidad de cotejar sus previsiones para los años 2000, 2005 y 2010 con la evolución real de la caja de las pensiones, y la posible influencia en el sistema previsional de sus prescripciones para atajar la crisis acrecientan su atractivo de cara al análisis.

Determinar el grado de acierto de las predicciones, su contribución a la percepción del riesgo de colapso de la Seguridad Social y sus eventuales efectos reflexivos nos permitirán retomar con nuevos elementos la polémica generada en los años '90 acerca de la viabilidad del sistema público y valorar si se había sobredimensionado el riesgo y, en tal caso, buscar la explicación del fallo predictivo. Confiamos en enriquecer así la comprensión del poder persuasivo de la predicción y de los procesos de definición de riesgos sociales en una coyuntura de desvalorización de las pensiones públicas, justificada en razón de la vaticinada incapacidad del fisco para sostenerlas.

\section{OBJETIVOS Y PROCEDIMIENTO}

Este trabajo pretende discernir la aportación de las predicciones difundidas en España a mediados de los años '90 a la percepción del riesgo de insolvencia de las pensiones públicas; y explorar las reacciones que hayan suscitado en sus destinatarios -autoridades y cotizantes de la Seguridad Social- y su impacto en el cumplimiento o incumplimiento de lo pronosticado.

Se parte de la hipótesis de que dichas predicciones, en línea con las precedentes del Banco Mundial y la OCDE, compartían una visión pesimista sobre el futuro del sistema de reparto junto con propuestas de reducción de la protección social y de promoción de las jubilaciones privadas.

El corpus de análisis lo integran cinco informes sobre el futuro de las pensiones españolas publicados entre 1995 y 1996, los primeros de su tipo en España: La reforma del sistema público de pensiones en España (Herce y Pérez Díaz, 1995); La Seguridad Social en el umbral del siglo XXI (Ministerio de Trabajo y Seguridad Social, 1996); El futuro de las pensiones en España: hacia un sistema mixto (Herce, Sosvilla Rivero, Castillo y Duce, 1996); Una propuesta de reformas del sistema de pensiones en España (Piñera y Weinstein, 1996); Pensiones y prestaciones de desempleo (Barea y González Páramo, 1996). Estudiaremos cada informe por separado, identificando su adscripción institucional, sus premisas, su objetivo manifiesto, su método y sus propuestas; y verificaremos su acierto predictivo con respecto a la trayectoria de la Tesorería de la Seguridad Social en los años posteriores. Por último, evaluaremos el seguimiento de sus recomendaciones por parte de las autoridades en lo tocante a la normativa del sistema de reparto y por parte de los afiliados a la Seguridad Social en cuanto a la suscripción de planes de pensiones.

\section{ANÁLISIS: PERFIL DE LOS INFORMES Y SUS PREDICCIONES}

\section{La reforma del sistema público de pensiones en España (1995)}

Autores y adscripción institucional: un equipo de economistas dirigido por José Antonio Herce y Víctor Pérez Díaz, financiados por el Servicio de Estudios de La Caixa. El primero es un economista vinculado a la Fundación de Estudios de Economía Aplicada (FEDEA) -centro de estudios de ideario liberal sostenido por bancos y cajas de ahorro españolas-; el segundo, un sociólogo que repetidas veces ha defendido la previsión individual (Pérez Díaz, 2000).

Objetivo: "mejorar la calidad de ese debate sobre el futuro de las pensiones" (Herce y Pérez Díaz, 1995, p. 9) con una finalidad persuasiva: "los electores no prestarán su apoyo en las urnas a esas recomendaciones si perciben que éstas les perjudican, y los partidos no les darán siquiera cobijo en sus programas, a no ser que (y ésta es una matización crucial) se introduzcan consideraciones suficientemente persuasivas para que a la vista de un 'interés común' se redefinan las premisas de los propios intereses a largo plazo de esos electores (...). Es a esta redefinición de los intereses a largo plazo a lo que puede contribuir el análisis de las estrategias de los actores y de la opinión pública" (Herce y Pérez Díaz, 1995, p. 12). Del efecto reflexivo perseguido se dice: "las «predicciones» desfavorables pueden resultar el mejor antídoto contra su propio cumplimiento, ya que cuanto más certeras y serenas, tanto más fácil será que los individuos de una sociedad, tomándolas como puntos de referencia para su reflexión y sus conversaciones, entiendan el problema y acepten enfrentarse a él" (Herce y Pérez Díaz, 1995, p. 11 y p. 12).

Método: Se realiza una proyección a treinta años de las tendencias actuales de crecimiento del PIB y del empleo combinada con proyecciones de la población española para 1991-2026 elaboradas por el Instituto de Economía, Geografía y Demografía del Consejo Su- 
perior de Investigaciones Científicas (CSIC), además de un sondeo de los actores sociales y políticos frente a un escenario hipotético de elección entre un sistema de reparto y otro de capitalización. Con los datos producidos se elabora una proyección macroeconómica acerca del efecto fiscal de cada una de las siguientes medidas: disminución de los puntos de pensión por año cotizado; aumento de la edad de retiro; aumento de la base reguladora de la prestación; penalización de la jubilación anticipada; y revalorización de los haberes inferior a la inflación.

Previsiones: Partiendo de un déficit del sistema de pensiones para 1996 estimado en el 2,16\% del PIB, se prevé que los pagos continuarán superando a los ingresos, llegándose a un déficit de 3,47\% el año 2010, un desequilibrio que comprometerá la viabilidad del sistema. La ratio cotizante / pensionista, que en 1995 era de 1,77, disminuirá a 1,68 en 2000, a 1,66 en 2005 y a 1,64 en 2010, una relación considerada insuficiente para garantizar pensiones adecuadas a las clases pasivas. El cálculo del impacto fiscal de las medidas propuestas indica que con cualquiera de ellas los déficit serán inferiores a los previstos: $-1,72 \%$ del PIB en caso de una revalorización de las pensiones inferior al IPC; o -3,06\% del PIB si disminuyesen los puntos de pensión por año cotizado. Del sondeo se destaca "la favorable disposición de una buena parte a que se potencie el componente privado del sistema de pensiones" (Herce y Pérez Díaz, 1995, p. 128).

Propuestas: Combinaciones de las cinco medidas mencionadas con incentivos fiscales a la contratación de pensiones privadas (Herce y Pérez Díaz, 1995, p. 126).

\section{La Seguridad Social en el umbral del siglo XXI (1995)}

Autores y adscripción institucional: Un colectivo de técnicos de la Dirección General de Planificación y Ordenación Económica de la Seguridad Social es el responsable de este trabajo que prolonga una previsión anterior sobre gastos e ingresos de la a Seguridad Social entre 1985 y 1993. Firma el prólogo el entonces ministro de Trabajo José A. Griñán, la prueba del aval institucional y político a este estudio cuyas previsiones aseguran la viabilidad del sistema público para 1995-2030.

Objetivos: De acuerdo con Griñán, prever el desenvolvimiento de la Seguridad Social con la finalidad de "adoptar, con tiempo suficiente, las decisiones necesarias para su adecuación a la realidad social y económica" (Ministerio de Trabajo y Seguridad Social, 1996, p. VIII), además de "presentar propuestas para encauzar adecuadamente su futuro" (Ministerio de Trabajo y Seguridad Social, 1996, p. X) y "consolidar un siste- ma de protección social que recibe un altísimo grado de consenso dentro de nuestra sociedad" (Ministerio de Trabajo y Seguridad Social, 1996, p. XI).

Método: Estudio económico-actuarial para el período 1995-2030 con métodos estadísticos y matemáticos empleados por el sector financiero-asegurador en la evaluación de riesgos, sobre la base de la proyección demográfica del Instituto Nacional de Estadística (INE) hasta el año 2020. Lo acompaña un análisis de sensibilidad (cálculo de los nuevos flujos de caja que se producirían al cambiar variables como inversión inicial, duración, ingresos, costes...) dirigido a prever cómo variarían los resultados del estudio anterior, de modificarse alguna de las hipótesis de partida tras la aplicación de medidas encaminadas a obtener ingresos adicionales o a reducir erogaciones.

Previsiones: Partiendo de la hipótesis de un incremento real del PIB del $2 \%$ anual, se estima que el gasto en pensiones subirá de un 9,06 \% del PIB en el año 1993 a un $9,83 \%$ en 2010 , y a $11,18 \%$ en 2030 . En el supuesto de un crecimiento de la riqueza nacional del 2,5\%, el gasto se situaría en el 9,23\% del PIB en 2010 y en $9,52 \%$ en 2030 . De no modificarse el régimen vigente, los desembolsos ocasionarán déficits equivalentes al $1 \%$ del PIB en el año 2000 y al 0,9 \% en 2010. A continuación, se simulan los escenarios de aplicación de cuatro reformas: aumento en un año de la edad de retiro; aumento en dos años de dicha edad; mayor proporcionalidad entre la cuantía de la jubilación y la de la invalidez; y subida de un punto del tipo de cotización. En función de cada medida el déficit en 2010 oscilaría entre 0,14 y 0,42 \% del PIB. Por último se calcula el costo para las arcas públicas del paso inmediato a un sistema de capitalización -los capitales de cobertura de los pasivos existentes a 1 de enero de 1995 sumados a la pérdida de los cotizaciones de los trabajadores, que aportarían a planes de pensiones- resultando que durante 1995/2010 el Estado debería destinar al pago de las jubilaciones adeudadas un equivalente a 2,11 veces el PIB de 1995, cifras que "ponen de manifiesto la inviabilidad técnica y económica de sustituir un sistema de reparto ya maduro por otro de capitalización, aparte de otras consideraciones negativas de carácter social" (Ministerio de Trabajo y Seguridad Social, 1996, p. 163).

Propuestas: Las simulaciones muestran que algunas de las medidas señaladas bastarían para consolidar el equilibrio del régimen contributivo, lo cual, se concluye, va en la línea de las recomendaciones del Pacto de Toledo ${ }^{2}$, proclives al perfeccionamiento paulatino del sistema de reparto y contrarias a su transformación radical (v. g. la implantación de la capitalización). 


\section{El futuro de las pensiones en España: hacia un sistema mixto (1996)}

Autores y adscripción institucional: J. A. Herce, S. Sosvilla Rivero, S. Castillo y R. Duce (expertos de FEDEA), con financiación del Servicio de Estudios de La Caixa, entidad que comercializa planes de pensiones.

Objetivo: Partiendo de las "insuficiencias" del Pacto de Toledo, el Servicio de Estudios de La Caixa pidió a FEDEA que "estudie los efectos y resultados de una de las posibles alternativas al sistema de reparto" (Herce, Sosvilla Rivero, Castillo y Duce, 1996, p. 6) con el fin de "aportar elementos de reflexión a un debate tan necesario como el de las pensiones" (Herce, Sosvilla Rivero, Castillo y Duce, 1996, p. 6). Y se añade: "realizar una aportación a esta nueva orientación del debate sobre la reforma de las pensiones sustanciando las razones por las que la transición (a un sistema mixto) puede ser deseable y explorando algunas de sus modalidades" (Herce, Sosvilla Rivero, Castillo y Duce, 1996, p. 9).

Método: Versión mejorada de la metodología usada por Herce y Pérez Díaz (1995) "como consecuencia de la actualización tanto de la base de datos del sistema español de pensiones como de las proyecciones de población, así como por la introducción de mejoras en la programación del modelo MODPENS utilizado en las simulaciones" (Herce y Pérez Díaz, 1995, p. 10). Se combina una proyección demográfica hasta el año 2050 sobre datos del Instituto de Economía, Geografía y Demografía del CSIC con otra macroeconómica de 22 variables relativas a los beneficiarios del sistema de pensiones, en un escenario sin cambios normativos. Con las cifras obtenidas se diseña un escenario de implantación de un sistema mixto en 1997, en el cual los cotizantes de entre 25 y 45 años de edad pueden trasladar la mitad de sus aportaciones a fondos privados; y en función del número de estos se elaboran distintas proyecciones sobre la cuantía de las pensiones resultantes y las cuentas de ambos sistemas con el horizonte del año 2050. Por último, un análisis de sensibilidad de los principales indicadores económicos calcula la rentabilidad futura de los planes de pensiones.

Previsiones: Déficit creciente de las pensiones públicas desde un montante equivalente a un 0,58\% del PIB en 1996 hasta alcanzar el 5,96\% en 2050. El deterioro financiero creará una deuda que llegará al $95,26 \%$ del PIB hacia 2050, un porcentaje que bajaría al $73,31 \%$ de iniciarse una transición gradual al sistema mixto. A los planes de pensiones se les pronostica una rentabilidad neta anual que va del 2,5 al 4,5\%, de los cuales aquellos con una rentabilidad de entre 3,5 y
4,5\% garantizarán cuantías superiores a las ofrecidas por el sistema de reparto. Se concluye que un sistema mixto permitirá reducir el déficit y aumentar las cantidades percibidas por los jubilados. Propuestas: Transición gradual a un sistema mixto basado en la capitalización obligatoria. Repárese en que mientras Herce y Pérez Díaz (1995) proponían la introducción de estímulos para una transición voluntaria en esa dirección, aquí se postula una mudanza total y coactiva.

\section{Una propuesta de reformas del sistema de pensiones en España (1996)}

Autores: Realizado por José Piñera y Alejandro Weinstein para el International Center for Pension Reform, un centro dedicado a la promoción de las pensiones privadas bajo la guía del propio Piñera, el ministro de Trabajo de la dictadura de Augusto Pinochet que impuso dichas pensiones a los trabajadores chilenos. El estudio lo patrocinó el Círculo de Empresarios, asociación consagrada a "la promoción de la libre empresa" en España con el apoyo de los bancos Santander, INGDirect, BBVA y Sabadell, entre otras entidades promotoras de planes de pensiones.

Objetivo: Analizar las condiciones de implantación, coste e implicaciones del sistema de capitalización en España.

Método: Para calcular el crecimiento poblacional se utilizó la proyección de 1994 del Instituto de Economía, Geografía y Demografía (CSIC) para el periodo 1991/2026; y para la evolución de la población económicamente activa se extrapolaron los antecedentes históricos. Luego se simuló una transición paulatina al sistema de capitalización con la tasa de cotización actual a la Seguridad Social.

Previsiones: Prevé la entrada en déficit del sistema de pensiones públicas a partir de 1996, fecha en la que los números rojos iniciarán una escalada hasta llegar a 5,49 billones de pesetas en 2025. En paralelo se acumulará una deuda que, sumados los intereses, equivaldrá al $56 \%$ de PIB en el año 2025. A continuación, se presenta un escenario de libre elección de planes de pensiones para mayores de 45 años, calculando los costos que le depararía al Estado la pérdida de sus cotizaciones y el ahorro que le supondría no pagar nuevas pensiones. Se concluye que el déficit causado por la transición gradual a la capitalización sería menor al que se produciría de no acometerse la reforma (estos cálculos buscan refutar a Barea y otros autores que denuncian lo gravoso que resultaría para las arcas públicas la implantación total de las pensiones privadas). 
Propuestas: Sustitución gradual del sistema de reparto por la capitalización individual.

\section{Pensiones y prestaciones de desempleo (1996)}

Autores y adscripción institucional: Un equipo interdisciplinar coordinado por los economistas José Barea y José Manuel González-Páramo, con financiación de la Fundación BBV del banco homónimo. El primer codirector, miembro del Comité Científico de dicha entidad, más tarde dirigió la Oficina Presupuestaria del gobierno de José M. a Aznar; el segundo ocupó cargos directivos en el Banco de España y el Banco Central Europeo (partidario de las pensiones privadas).

Objetivo: "analizar con rigor y perspectiva interdisciplinar los modelos de aseguramiento de la inactividad laboral en España, con la aspiración de alentar la reflexión colectiva acerca de las modificaciones y las reformas necesarias" (Barea y González Páramo, 1996, p. 14)

Método: "Modelo estilizado de simulación que integra los factores económicos y demográficos que van a condicionar el futuro" (Barea y González Páramo, 1996, p. 213), apoyado en tres factores estratégicos: demográficos, económicos y normativos. Para la evolución económica se apoya en las proyecciones del Centro de Predicción Económica de la Universidad Autónoma de Madrid para el periodo 1995-2000 (prolongadas con modificaciones hasta el año 2020); en lo demográfico utiliza las estimaciones del INE hasta 2020; y para lo normativo maneja un supuesto de estabilidad legislativa.

Previsiones: El déficit de las pensiones registrado en 1993 se duplicará en el 2000, llegando al 1,65\% del PIB en 2005 y al 1,8\% en 2020. Se sigue que el sistema de reparto, de no modificarse, se tornará inviable a principios del siglo XX (para estos autores, el umbral de inviabilidad lo marca el límite de déficit público del 3\% del PIB fijado por el Tratado de Maastricht). EI tránsito brusco a la capitalización le costaría a la Seguridad Social un montante equivalente a 2,5 veces el PIB de 1996, derivado del pago de haberes adeudados a los beneficiarios del sistema extinguido y a la pérdida de la totalidad de sus cotizantes.

Propuestas: Descartado el recurso al aumento de impuestos o de cotizaciones laborales por su impacto negativo en la competitividad empresarial, así como la adopción total de un sistema de capitalización -por los gastos que acarrearía al fisco-, se aconseja aplicar estrictamente el criterio de proporcionalidad entre cotizaciones y prestaciones, y redoblar la lucha contra el fraude.

\section{GRADO DE CUMPLIMIENTO DE LAS PREDICCIONES}

En la tabla 1 se comparan las proyecciones de los cinco estudios con la evolución real de los desembolsos en pensiones públicas:

Vemos que todas las predicciones se incumplieron, ya que auguraban déficits cuando lo que hubo fueron superávits (las que más se aproximaron a la realidad fueron las del Ministerio de Trabajo). Ninguna imaginó los excedentes que, además de compensar el saldo negativo de las prestaciones sanitarias, sostuvieron el crecimiento del Fondo de Reserva de la Seguridad Social $^{3}$, que a finales de 2010 ascendía a más de 64.375 millones de euros (6,11\% del PIB de 2009). Herce, Sosvilla Rivero, Castillo y Duce previeron una ratio afiliados / pensionistas declinante (1,64 en 2010) cuando ocurrió lo contrario (2,56 a finales de 2010). Y si bien las predicciones sobre el costo fiscal del tránsito a un sistema de capitalización no pudieron verificarse, pues este escenario no se materializó, los yerros en horizontes tan cercanos restan credibilidad a sus proyecciones a 50 años.

Un párrafo aparte merece el fallido pronóstico de Herce, Sosvilla Rivero, Castillo y Duce sobre la rentabi-

Tabla 1. Déficits previstos de las pensiones públicas y evolución real de sus finanzas (expresados en porcentajes del PIB)

\begin{tabular}{lccc}
\hline Fuente & Año 2000 & Año 2005 & Año 2010 \\
\hline Herce y Pérez Díaz & $-2,6 \%$ & $-2,75 \%$ & $-2,94 \%$ \\
\hline Ministerio de Trabajo & $-0,1 \%$ & $-0,40 \%$ & $-0,90 \%$ \\
\hline Herce et al. & $-1,37 \%$ & $-1,8 \%$ & $-2,15 \%$ \\
\hline Barea y González Páramo & $-1,6 \%$ & $-1,65 \%$ & $-1,42 \%$ \\
\hline Piñera y Weinstein & $-0,42 \%$ & $-0,79 \%$ & $\mathbf{0 , 2 2 \%}$ \\
\hline Evolución real & $\mathbf{0 , 3 3 \%}$ & $\mathbf{0 , 3 0 \%}$ & \\
\hline
\end{tabular}

Fuente: elaboración propia con datos de los autores citados y de la Seguridad Social. 
lidad de los planes de pensiones, estimada entre 2,5 y 4,5\% anual (inflación descontada) para el período 1995/2050. El estudio de 532 fondos de pensiones realizado por Fernández, Aguirreamalloa y Corres (2012) enseña que en el lapso 2000/2011 la rentabilidad de los bonos del Estado a diez años fue de 5,13\% anual, pero solo dos fondos mejoraron esta cota; apenas 27 superaron la inflación promedio y 191 tuvieron rentabilidad negativa. En breve: el 99,6\% de los planes rindió menos que los bonos -una inversión de alta seguridad y baja rentabilidad-, y el $95 \%$ menos que la inflación, en claro perjuicio de sus suscriptores, dando un mentís rotundo a quienes prometían en todos los casos una rentabilidad superior al alza de los precios.

¿Razones de los fallos? Entre las que hemos podido identificar figuran las siguientes: datos iniciales erróneos -sobreestimación del déficit de las pensiones en 1995 por parte de Herce y Pérez Díaz, que lo situaron en el 2,16\% del PIB cuando fue del 0,23\%-; subestimación de la inmigración -entre 1996 y 2010, los extranjeros censados por el INE pasaron de 542.314 a 5.747.734- y de la caída del desempleo, factores que en 2007 llevaron el número de afiliados a la Seguridad Social al máximo histórico de 19,4 millones; y la desviación de las proyecciones del CSIC, que vaticinaron 41,3 millones de residentes para 2015, cuando en 2012 el INE ya contabilizaba 46.196.278.

\section{EFECTOS DE LAS PREDICCIONES}

Resulta difícil, si no imposible, imputar efectos directos a las predicciones debido a la infinidad de variables en liza y a la diversidad de actores que en el lapso estudiado compitieron por incidir en el régimen de las pensiones estatales. Sí se puede descartar que fungiesen de "profecías auto-cumplidas", pues el deterioro financiero anunciado no tuvo lugar (en verdad, la obligatoriedad del sistema de reparto imposibilitaba tal eventualidad: ningún cotizante tenía derecho a retirar sus aportaciones de la Seguridad Social). Tampoco actuaron de "profecías suicidas", ya que en la bonanza fiscal influyeron variables independientes como la inmigración y el aumento de la ocupación. En definitiva, cualesquiera hayan sido los efectos de las predicciones, estos no repercutieron en las cuentas de una institución que hasta 2012 no atravesó cambios sustanciales.

El balance presenta otro aspecto, de considerarse las recomendaciones ligadas a las predicciones. En el periodo 1995-2012, las autoridades económicas concedieron generosas bonificaciones fiscales a las pensiones privadas -medida aconsejada por Herce y Pérez Díaz- retrasaron la edad de jubilación a los 67 años -opción barajada por Herce y Pérez Díaz y el Ministerio de Trabajo- y ampliaron a 25 años el periodo cotizado para el cálculo de la base reguladora -propuesta de Herce, Sosvilla Rivero, Castillo y Duce. Que tales actuaciones fueran exigidas igualmente por la patronal española, economistas y organismos internacionales impide atribuirlas exclusivamente al poder persuasivo de las predicciones, aunque sin duda estas sirvieron de caja de resonancia de tales demandas. Pero hay una recomendación cuyo seguimiento dependía en última instancia del comportamiento del público: la suscripción de planes de pensiones aconsejada por Herce y Pérez Díaz, Herce, Sosvilla Rivero, Castillo y Duce, y por Piñera y Weinstein. En la tabla 2 se observa que, tras la difusión de las predicciones, el número de suscriptores y el patrimonio acumulado crecieron sustancialmente.

Entre 1995 y 2000 el número de titulares de planes casi se triplicó, y entre 2000 y 2011 se duplicó. Entre 1995 y 2011 los activos gestionados por bancos y fondos de inversiones se multiplicaron por un factor de trece. ¿Cómo se explica este crecimiento exponencial? Sin duda intervinieron dos factores de gran peso: los beneficios fiscales que desde 1988 hicieron de los planes un apetecible instrumento de ahorro para los sectores de ingresos medio-altos (ley 40/1988, ley 35/2006, o ley foral $3 / 1988$ de Navarra) y la desconfianza en las jubilaciones públicas alentada por las predicciones ${ }^{4}$. Aunque resulta imposible determinar la contribución precisa de cada factor, es obvio que ambos se complementaron y reforzaron mutuamente: las visiones de bancarrota estatal predispusieron a una franja de la población a la búsqueda de alternativas a las pensiones públicas; el acicate material de las desgravaciones orientó esa búsqueda en el sentido deseado.

Tabla 2. Evolución de los planes privados de pensiones en España (1995-2011)

\begin{tabular}{lllll}
\hline Año & $\mathbf{1 9 9 5}$ & $\mathbf{2 0 0 0}$ & $\mathbf{2 0 0 5}$ & $\mathbf{2 0 1 1}$ (marzo) \\
\hline Número de partícipes & 1.796 .084 & 4.938 .828 & 9.290 .313 & 10.720 .000 \\
\hline Patrimonio en millones de euros & $6.306,3$ & $21.494,2$ & $86.588,0$ & 84.989 \\
\hline
\end{tabular}

Fuente: Asociación de Instituciones de Inversión Colectiva y Fondos de Pensiones. 
En síntesis: las predicciones no fungieron de "profecías sociales" puesto que no causaron acciones que desembocasen en el advenimiento o frustración de lo pronosticado. Pero no es descabellado atribuirles cierta responsabilidad en la implantación de algunas de las propuestas formuladas por sus autores; propuestas que, de todos modos, no afectaron a las cuentas públicas al menos hasta el año 2010. Mucho más obvia, en cambio, parece su contribución a la formación del clima de opinión propicio a la expansión de las pensiones privadas registrada en el lapso estudiado.

\section{DISCUSIÓN DE LOS RESULTADOS}

¿Se deduce del fallo de las predicciones que estas exageraron el riesgo de colapso de la Seguridad Social? En la medida en que su yerro no fue el corolario de procesos reflexivos desencadenados por ellas mismas sino el resultado de las dinámicas endógenas del sistema, se hace evidente que el riesgo fue sobrestimado. Surge entonces la cuestión: ¿respondía la sobreestimación al designio de inculcar en la opinión pública la necesidad de cambios en la política social mediante el bosquejo de escenarios calamitosos? No busquemos la respuesta en lo manifestado expresamente por los autores. Aunque todos declararon su intención de atajar los males previstos alertando del problema en ciernes, ninguno, a excepción de Herce y Pérez Díaz, reconoció la utilidad de los pronósticos alarmistas en pos de ese objetivo. Más productivo resulta buscar una respuesta en los premisas y sofismas presentes en la mayoría de los trabajos que, pese a su pretendida imparcialidad y naturaleza técnica, les predispusieron a magnificar el riesgo de las jubilaciones.

Repárese en la repetida afirmación de que el sistema de reparto se halla en aprietos ("[l]a financiación de las pensiones es una de las áreas problemáticas más importantes de la sociedad actual", Barea y Gonzalez Páramo, 1996, p. 14), aseveración que entraña una petición de principio, pues precisamente lo que las predicciones debían dilucidar era si tal problema se produciría. Otro sesgo lo pone la presunción de un "techo" en el gasto en pensiones -el porcentaje del PIB que marca el umbral "inasumible" por el Estado (Barea y González Páramo, 1996)- supuesto que, aparte de ignorar a países como Holanda o Luxemburgo, que destinan a esa partida el $15 \%$ de su PIB sin arruinarse por ello, soslaya la ausencia de "una teoría que nos permita señalar, no como ciudadanos sino como economistas, cuándo un determinado gasto es o no asumible por el sector público. En efecto, ¿qué significa «no asumible»? ¿Significa que su superación o su 'transgresión' desencadenaría una secuencia de acontecimientos catastróficos?" (Esteve Mora y Muñoz de Bustillo Llorente, 2004, p. 301). Lejos de ser un dato de la realidad, el "techo" se fundamenta en la opinión de que cuanto menor sea el gasto público, mejor. Otro tanto puede decirse del fatalismo demográfico que impregna muchos informes ("A medio plazo, los gastos futuros de pensiones están ya determinados por la estructura demográfica actual”, Barea y González Páramo, 1996, p. 238).

Menos tendenciosas en apariencia, pero esencialmente sesgadas, son ciertas definiciones del objeto de la predicción. Véase la consideración del seguro colectivo como un asunto puramente contable; un pase de manos conceptual que difumina la razón de ser de esa institución: garantizar la equidad y otros valores sociales ausentes en previsiones condicionadas por su adhesión al precepto del equilibrio fiscal. O la equiparación falaz del sistema de reparto a una empresa, implícita en la afirmación de que "está abocado a la quiebra" (Piñera y Weinstein, 1996, p. 11), cuando ningún Estado quiebra como una firma comercial, con convocatoria de acreedores y liquidación de activos, aunque como metáfora sirve para asustar al público con la pérdida de sus derechos jubilatorios.

Política e ideológicamente connotadas son tanto las soluciones elegidas para atajar el riesgo expuesto como el descarte de ciertas alternativas. La apuesta por las pensiones privadas reconduce el manejo de un riesgo eminentemente social a las salidas individuales caras al neoliberalismo. Con todo, cabría esperar que sus avalistas, si es que realmente pretendían ayudar a invertir con conocimiento de causa, tuvieran en cuenta las eventualidades negativas inherentes a dicha opción (derrumbe bursátil, inflación, comisiones de gestión, quiebra de los fondos) ${ }^{5}$. Por el contrario, llama la atención que Herce y Pérez Díaz y Piñera y Weinstein "no hayan desarrollado en sus trabajos el cálculo de la rentabilidad y fiabilidad de los planes privados de inversión y pensiones. No cuestionan la viabilidad de las pensiones privadas, partiendo de la premisa de que son rentables y podrá mantenerse el sistema" (Iglesias Fernández, 2010 p. 48). E incluso quienes hicieron cálculos de ese tipo, como Herce, Sosvilla Rivero, Castillo y Duce, que auguraron rentabilidades tan fallidas como el resto de sus previsiones, contribuyeron a minimizar el riesgo de esas inversiones.

Preconcepciones similares justifican el rechazo de otras opciones, lo que restringe de antemano las soluciones disponibles. Tal el caso de la negativa a "que los déficits se financien con los impuestos generales del 
Estado" (Barea y González Páramo, 1996, p. 227). Al rechazar el aumento de la presión fiscal -la vía escogida por Japón y Dinamarca para solventar los desfases entre ingresos y gastos del sistema público- se torna más fácil calificar de insostenibles los futuros desequilibrios del modelo de reparto y defender los recortes o la privatización como la única salida.

La exageración del riesgo de las pensiones públicas y el silencio sobre el riesgo de las privadas no se puede desligar de la adscripción institucional de los informes. Cuatro tenían por autores a expertos de think tanks partidarios de la reducción del gasto social o de entidades con intereses en el mercado de pensiones ${ }^{6}$; el quinto, a técnicos de un ministerio comprometido con el modelo público. No es casual que los cuatro primeros vaticinasen el apocalipsis fiscal ni que el restante previera escenarios tranquilizadores: la percepción aguda del riesgo del sistema de reparto era tan funcional a las políticas de recortes de prestaciones y su sustitución / complementación por la capitalización individual, como lo era la percepción de un riesgo bajo la defensa del modelo vigente.

¿Es legítimo concluir que los fallos se debieron a sesgos políticos e ideológicos? No enteramente. Que todas las estimaciones hayan errado empleando métodos similares pone de relieve la escasa fiabilidad de las predicciones, al margen del color ideológico de sus autores. Quizás los errores se hubieran atenuado de haberse manejado mayor número de variables y escenarios y una metodología más compleja que la extrapolación de unas pocas tendencias, pero no se habrían evitado: no hay matemática capaz de anticipar fenómenos imprevisibles como los migratorios, por ejemplo. El problema de fondo radica en la estructura de la predicción, muy vulnerable a las sorpresas que deparan los procesos dinámicos no lineales. De aquí que se deba utilizar con la máxima cautela, a sabiendas de que su carácter provisional obligará a corregirla periódicamente.

De esa provisionalidad no se dice nada en la mayoría de los informes. Se entiende el porqué: hablar de un "problema de las pensiones" da por sentada la existencia de un riesgo que posteriormente será confirmado mediante la presentación de un "inexorable"7 escenario calamitoso. Al exagerar la magnitud de la crisis en ciernes con la perspectiva de déficits incontrolables, las predicciones allanaron el camino a las recetas a las que venían atadas, como la promoción de las pensiones privadas ${ }^{8}$. Estas tuvieron tal expansión en los años siguientes que, a juicio de Iglesias Fernández (2010), "el resultado que las entidades financieras buscaban con estos anuncios catastrofis- tas se cumplía. El dinero dedicado a estos estudios, por mucho que nos parezca comparado con nuestros ingresos, es muy rentable. Los fabricantes del miedo pueden felicitarse por este éxito" (p. 46). Ciertamente, el poder persuasivo de las predicciones habría sido menor de haberse admitido con franqueza su naturaleza especulativa y su propensión al error.

En resumidas cuentas, aparte de su incidencia en el mercado de pensiones, el efecto más visible de las predicciones ha sido el de instalar en la opinión pública un problema atado a determinadas soluciones, amén de engendrar una progenie de vaticinios y encuestas ideadas para atizar el debate sobre la "cuestión" de las pensiones públicas, su "incierta” financiación y las ventajas de la capitalización ${ }^{9}$. Esta labor persuasiva resulta inevitable en países democráticos donde la privatización no se puede imponer manu militari como en el Chile de Pinochet. No es sencillo destruir la confianza social en un régimen que viene garantizando retiros relativamente desahogados desde hace treinta años; exige construir el "problema", convencer a la sociedad de que una de las instituciones más saneadas del Estado va camino al colapso; una vez establecida esa percepción, la banca y sus economistas proveerán el remedio. En ese marco entran a jugar las predicciones que, en un movimiento inverso al del especulador bursátil que propaga bulos para que los accionistas malvendan sus valores (Kapferer, 1990), inducen a los afiliados a la Seguridad Social a suscribir planes de pensiones. Ahora bien, para influir en la clientela potencial de las jubilaciones privadas deben difundirse masivamente. Un requisito cumplido por los casos estudiados, vista su repercusión en la prensa ${ }^{10}$. Como observa Duval (2002) sobre los informes catastrofistas relativos al estado de bienestar francés, las dinámicas mediáticas propician que tales anuncios, hábilmente comunicados en ruedas de prensa y otras iniciativas de relaciones públicas, consagren en la agenda pública el tópico del "agujero de la Seguridad Social" incluso contra las reticencias de periodistas críticos.

\section{CONCLUSIONES}

El análisis ha mostrado cómo la mayoría de las predicciones estudiadas planteó una perspectiva verosímil de crisis financiera de la Seguridad Social en el corto y el mediano plazo, definiendo en términos locales el riesgo de colapso anunciado por el Banco Mundial y la OCDE. A posteriori, sus estimaciones se demostraron exageradas, en parte debido a los sesgos y premisas de su diseño y en parte a las limitaciones intrínsecas de toda proyección económico-demográfica. 
Los datos confirman mayormente la hipótesis manejada: cuatro de las predicciones, con sus visiones pesimistas del envejecimiento poblacional vinculadas a horizontes de déficits estatales y propuestas de disminución de la protección social, encajan en los parámetros del "terrorismo demográfico"; solo la quinta se apartó de esa pauta al minimizar la posibilidad de un colapso de la caja de pensiones; una diferencia que corre paralela a sus diferentes adscripciones institucionales: entidades financieras y centros de estudios neoliberales en el primer caso, la Seguridad Social en el segundo. Fue parcial el refrendo de la hipótesis de que las recomendaciones incluidas en los informes favorecían la expansión de la capitalización en detrimento del reparto, pues solo tres de estos apuntaban en esa dirección, mientras que el informe de Trabajo apostaba por seguir las directrices fijadas por el Pacto de Toledo, y el de Barea y González Páramo se inclinaba por recortar las prestaciones y endurecer sus requisitos dentro de la arquitectura institucional vigente.

Se perfila así un cuadro más complejo del previsto: de un lado, una mayoría maximizaba el riesgo de las pensiones; del otro, una minoría los minimizaba. A su vez aquella mayoría se dividía entre abanderados del equilibrio fiscal y promotores del mercado de pensiones. Los primeros urdían escenarios que cuestionaban el sistema de capitalización, mientras los segundos pergeñaban proyecciones que afirmaban sus ventajas. En breve: los augurios sobre la Seguridad Social sirvieron de armas a las elites políticas y económicas en su pugna por el control del estado de bienestar.

En cuanto a la relación de los vaticinios con la reflexividad, el hecho de que no fungiesen de profecías sociales no les impidió actuar sobre la confianza pública en el sistema de reparto. Dado que el acierto de sus prognosis solo se determinaría años más tarde, en el corto plazo sus autores se aprovecharon de la probada eficacia de la categoría de riesgo para legitimar o desacreditar determinadas políticas o instituciones (Douglas, 1990, p. 5). Las dudas suscitadas en la opinión pública acerca de la solvencia de la Seguridad Social y el auge de las jubilaciones privadas hablan de la eficacia de una categoría de predicciones reflexivas distinta de las teorizadas por Merton.

En los últimos años hemos visto que los errores predictivos no han desanimado a las Casandras del déficit, las cuales, a la usanza de los antiguos profetas, reaccionan a sus yerros postergando la fecha del desastre ${ }^{11}$. Nada de irracional hay en su proceder, pues con él consiguen un objetivo preciso: tener a los trabajadores en permanente zozobra acerca de su futuro, y con ello alimentar un sentimiento generalizado de inseguridad que disciplina la mano de obra y favorece el mercado del seguro, al precio de una incertidumbre psíquicamente desestabilizadora y socialmente corrosiva. Por añadidura, muy pocos contradictores les salen al cruce. Que apenas uno de los informes examinados abogase por el reparto evidencia la actitud defensiva de los partidarios del estado de bienestar ante la avalancha de vaticinios pesimistas que, en línea con el discurso económico imperante, reiteran el falaz dilema entre su desmantelamiento o la ruina nacional. Tal pasividad recuerda lo referido por Lakoff (2007) acerca de la parálisis del progresismo frente a la comunicación política neoliberal. En España desde hace dos décadas los conservadores mantienen la ofensiva en una "guerra de los futuros posibles" en la que, armados de predicciones y encuestas, buscan convencer a la sociedad de la inevitabilidad de los escenarios que presentan. La sociología, conocedora del poder de las representaciones del porvenir y de la matriz sociocultural del riesgo, tiene mucho que decir en la batalla colectiva por definir los futuros de los que se debe pensar y opinar; queda a sus practicantes optar por asistir a los "fabricantes de miedo" o sumarse a su crítica ${ }^{12}$.

\section{NOTAS}

1 "Si la predicción se formula es porque es relevante para alguien y ese alguien alterará su conducta en función de la predicción, de modo que la reflexividad queda garantizada bien por el lado del actor, bien por la vía indirecta del sujeto predictor" (Lamo de Espinosa, 1988, p. 53).

2 Acuerdo parlamentario del 6 de abril de 1995, en el cual se fijaron las actuaciones y modificaciones que había que in- troducir en el régimen contributivo (ej. tránsito de un sistema de reparto puro a uno en el que se cobre en función de lo aportado, y el refuerzo del "sistema complementario" privado, voluntario y no sustitutivo de las contribuciones obligatorias).

3 El fondo fue creado en 1995 con el objetivo de destinar los excedentes de la Tesorería de la Seguridad Social a atender las necesidades futuras en el pago de las prestaciones contributivas originadas por desviaciones entre ingresos $y$ gastos.

4 Del calado de ese temor da fe el Barómetro del Centro de Investigaciones Sociológicas de marzo de 2011 sobre la reforma de las pensiones promovida por el gobierno de José Luis Rodríguez Zapatero: el 46,9\% de los encuestados se decantó por el enunciado: "no resuelve el problema de las pensiones y éste re- 
aparecerá en los próximos años", y solo el 15,3\% acordó con la aseveración: “La reforma no era necesaria porque el funcionamiento del sistema de pensiones no corría realmente ningún peligro" (Centro de Investigaciones Sociológicas, Estudio no 2864, p. 15 y p. 16).

5 Los fondos de pensiones sí quiebran. As ocurrió en Estados Unidos tras la crisis de 1929 y en 1974, cuando el gobierno federal tuvo que establecer la Pension Benefit Guaranty Corp. para asegurar una renta mínima a los afectados. Y en el Reino Unido, el Pension Protection Fund se ha hecho cargo de 631.000 suscriptores de fondos insolventes.

6 La sintonía de Herce, Sosvilla Rivero, Castillo y Duce (1996) con los intereses de sus patrocinadores se palpa en su declaración: "Hay que ver el desarrollo de la capitalización de pensiones en nuestros países como una oportunidad estratégica para el sector financiero" (p. 93).

7 El demógrafo Julio Pérez Díaz atribuye esa inexorabilidad a "un uso abusivo y decimonónico de la demografía. Se hace "contabilidad" de personas, se proyectan los stocks poblacionales $y$ se introducen los datos en modelos que inevitablemente arrojan resultados catastróficos (...) Como la evolución demográfica parece inexorable, el envejecimiento conduce a la inevitable conclusión de que 'hay que recortar'. Este uso resulta de un requisito metodológico ignorado: las proyecciones resultan de las tendencias previstas si, y sólo si,

\section{BIBLIOGRAFÍA}

Balmaseda, M., Melguizo, A. y Taguas, D (2006). Las reformas necesarias en el sistema de pensiones contributivas en España. Moneda y Crédito, 222, pp. 313-340.

Barea, J. y González Páramo, J. M. (eds.) (1996). Pensiones y prestaciones por desempleo. Madrid: Fundación BBV.

Beck, U. (1996). Risk society and the provident state. En: Lash, S., Szerzynski, B. y Wynne, B. (coords.). Risk, Environment and Modernity. Towards a new Ecology. London: SAGE, pp. 27-43.

Beck, U. (1998). La sociedad del riesgo: hacia una nueva modernidad. Barcelona: Paidos.

Bradbury, J. A. (1989). The policy implications of differing concepts of risk. Science, Technology \& Human Values, 14, 4, pp. 380-399. https://doi. org/10.1177/016224398901400404 'en igualdad del resto de condiciones' (...) El "resto de condiciones" que cambian junto a la pirámide de población puede ser descrita como una revolución productiva, sumamente favorable a la sostenibilidad futura de un sistema de reparto" (2011, p. 2 y ss.)

8 Que la banca tiene mucho que ganar con los planes de pensiones lo prueba el estudio de Fernández et al.: las elevadas comisiones cobradas por su gestión -entre 1,5 y $2,5 \%$ del patrimonio invertido- le reportaron 563 millones de euros en el año 2002, y en 2008, 1.013 millones. No sorprende su empeño en seguir costeando la industria predictiva.

9 Las encuestas sobre pensiones destinadas a su publicación en los medios les sirven a las entidades financieras para crear el estado de opinión deseado. Ver la de Pérez Díaz, Álvarez-Miranda y Chuliá (1997) o la confeccionada por Villafañe y Asociados para Mapfre Vida en 2011, en la que se proclama que "el porcentaje de ciudadanos que cree que el futuro de las pensiones no está garantizado se elevó respecto del año anterior en casi 10 puntos, hasta el 70\%" (ABC, 31/05/2011).

10 La afinidad de la prensa económica con los predictores fue reconocida por Herce y Pérez Díaz (1995): “los diarios económicos con mayor tirada, cuya preocupación continuada por las cuestiones del déficit público y la convergencia europea les lleva a contemplar más críticamente el actual sistema público de pensiones (p. 86)

Callahan, D. (1987). Setting Limits: Medical Goals in an Aging Society. New York: Simon \& Schuster.

Candel Sanmartín, M. (ed.) (1988). Tratados de lógica (Órganon). Aristóteles (vol. II). Madrid: Gredos.

Castel, R. (2004). La inseguridad social ¿Qué es estar protegido? Buenos Aires: Manantial.

Douglas, M. (1990). Risk and Blame. London: Routledge.

Duval, J. (2002). Une reforme symbolique de la Sécurité sociale. Les médias et "le trou de la Sécu". Actes de la Recherche en Sciences Sociales, 143, pp. 53-67. Disponible en http://www.persee.fr/doc/arss_03355322_2002_num_143_1_2855

Esteve Mora, F. y Muñoz de Bustillo Llorente, R. (2004). Mitos y falacias populares
11 Herce (2000) desplazó la fecha crítica al año 2050, pronosticando un desequilibrio equivalente al $6 \%$ del PIB. $Y$ Balmaseda, Melguizo y Taguas (2006) auguraron déficits para 2011 (cosa que tampoco se cumplió) y un gasto en pensiones del 13,5/24,0\% del PIB para 2059, concluyendo que: "alcanzado el equilibrio del sistema, debería permitirse la introducción de una parte de capitalización individual y gestión privada" (p. 20).

12 Al momento de la publicación de este artículo, se podría interpretar que el déficit actual de la Seguridad Social española da cierta razón (con retraso) a los vaticinios criticados en sus páginas. No disponemos de espacio para cuestionar esa impresión; solo añadir que en el desequilibrio poco ha tenido que ver la demografía, la variable determinante en dichos pronósticos. Lo decisivo ha sido la política económica con medidas tales como el abaratamiento de los despidos, la reducción de salarios y vacantes en la administración pública y las bonificaciones de los nuevos contratos laborales, además de la caída salarial en el sector privado favorecida por el temor al paro y las prejubilaciones masivas con cargo a la caja de las pensiones. Estas actuaciones, justificadas en aras de la competitividad y la reducción del gasto público, fueron tomadas a sabiendas de su impacto negativo en las cuentas de la Seguridad Social (i.e. reducción del número de cotizantes y de la cuantía de las cotizaciones). Ninguna fatalidad demográfica, en definitiva; y sí decisiones políticas de un signo preciso.

en el debate acerca de los sistemas de pensiones. Estudios de Economía Aplicada, 22, 2, pp. 289-316.

Ewald, F. (1986) L'État providence. París: Grasset.

Fernández, P., Aguirreamalloa, J. y Corres, L. (2012). Rentabilidad de los fondos de pensiones en España, 2001-2011. Documento de Investigación DI-947. Febrero 2012. IESE, Universidad de Navarra. [En línea]. Disponible en http://www.iese. edu/research/pdfs/DI-0947.pdf

Gee, E. y Gutman, G. (eds.) (2000). The overselling of Population Aging: Apocaliptic Demography, Intergenerational Challenges and Social Policy. Ontario: Oxford University Press.

Herce, J. A. (2001). La privatización de las pensiones en España. Documento de 
trabajo de FEDEA. Fundación de Estudios de Economía Aplicada. [En línea]. Disponible en: http://documentos.fedea.net/pubs/dt/2001/dt-2001-01.pdf

Herce, J. A. y Meseguer, J. A. (2000). La reforma de las pensiones ante la revisión del Pacto de Toledo. Barcelona: Servicio de Estudios La Caixa. Disponible en: http://www.caixabankresearch.com/ documents/10180/54279/ee19_esp.pdf

Herce, J. A. y Pérez-Díaz, V. (dirs.) (1995). La reforma del sistema público de pensiones en España. Barcelona: Servicio de Estudios La Caixa. Disponible en: http:// www.caixabankresearch.com/documents/10180/54279/ee04_esp.pdf

Herce, J. A., Sosvilla Rivero, S., Castillo, S. y Duce, R. (1996). El futuro de las pensiones en España: hacia un sistema mixto. Barcelona: Servicio de Estudios La Caixa. Disponible en: http:// www.caixabankresearch.com/documents/10180/54279/ee08_esp.pdf

Iglesias Fernández, J. (2010). Desde las entidades financieras (bancos, cajas y mutuas de seguros), un ataque permanente al sistema público de pensiones. En: Etxezarreta, M., Iglesias Fernández, J. Idoate, E. y Junyent Tarrida, J. Sin pensiones públicas, ¿qué futuro?, pp. 35-52. [En línea]. Disponible en: http:// www.mirenetxezarreta.es/wp-content/ uploads/2012/05/Libro-Pensiones1.pdf

Jerit, J. y Barabas, J. (2006). Bankrupt Rethoric. How Misleading Information Affects Knowledge about Social Security. Public Opinion Quaterly, 70, 3, pp. 278-303. https://doi.org/10.1093/poq/nfl010

Kapferer, J. N. (1990). La rumeur en Bourse. Communications, 52, 1, pp.61-84. https:// doi.org/10.3406/comm.1990.1783

Kennedy, G. (2009). Adam Smith and the Invisible Hand: From Metaphor to Myth. Economical Journal Watch, 6, 2, pp. 239-263.

Lakoff, G. (2007). No pienses en un elefante: lenguaje y debate político. Madrid: Editorial Complutense.

Lamo de Espinosa, E. (1988). Predicción, reflexividad y transparencia: la ciencia social como autoanálisis colectivo. REIS. Revista Española de Investigaciones Sociológicas, 43, pp. 43-74. https://doi. org/10.2307/40183343

Luhmann, N. (1992). El futuro no puede empezar: estructuras temporales en la sociedad moderna. En: Ramos Torre, R. (ed.) Tiempo y sociedad. Madrid: CIS / Siglo XXI, pp. 161-182.

Luhmann, N. (2006). Sociología del riesgo. México D F: Universidad Iberoamericana.
Lupton, D. (1999). Risk and Sociocultural Theory: New Directions and Perspectives. Cambridge: Cambridge University Press. https:// doi.org/10.1017/CBO9780511520778

Macció, G. y Unión Internacional para el Estudio Científico de la Población - UIECP (1985). Diccionario demográfico multilingüe. Lieja: Ordina.

Markert, J. (2005). The Malthusian fallacy: Prophecies of doom and the crisis of Social Security. The Social Science Journal, 42, pp. 555-568. https://doi. org/10.1016/j.soscij.2005.09.009

Martínez Noval, L. y Sáenz de Jubera Álvarez, P. (2009). El futuro ya no es lo que era. Las proyecciones de la Seguridad Social. Revista del Ministerio de Trabajo e Inmigración, 79, pp. 13-43.

Merton, R. (1948). The Self-Fulfilling Prophecy. The Antioch Review, 8, 2, pp. 193-210. https://doi.org/10.2307/4609267

Ministerio de Trabajo y Seguridad Social (1996). La Seguridad Social en el umbral del siglo XXI: estudio económico-actuarial. Madrid: Centro de Publicaciones del Ministerio de Trabajo y Seguridad Social.

O' Malley, P. (1996). Risk and Responsability. En: Barry, A., Osborne, T. y Rose, N. (eds.) Foucault and Political Reason: Liberalism, neo-liberalism and rationalities of government. London: University of Chicago Press, pp. 189-207.

Palacios, R. (1994). Averting the Old Age Crisis. Worl Bank Policy Research Working Paper. [En línea]. Disponible en: https:// core.ac.uk/download/pdf/6615707.pdf

Pérez Díaz, J. (2011). II. Los factores demográficos. En VV. AA. La reforma de las pensiones y su incidencia en el sector público. Cemical, pp. 15-19. Disponible en: https://www1.diba.cat/uliep/ pdf/53468.pdf

Pérez Díaz, V. (2000). Escuchar a la sociedad: ¿es el pacto de Toledo una respuesta? En: Herce, J. A. y Huerta de Soto, J. (coords.) Perspectivas del estado del bienestar: devolver responsabilidad a los individuos, aumentar las opciones. Fundación para el Análisis y los Estados Sociales, pp. 53-64. Disponible en: https://es.scribd.com/doc/303602730/ Perspectivas-del-Estado-del-BienestarDevolver-responsabilidad-a-los-individuos-aumentar-las-opciones-JoseAntonio-Herce-y-Jesus-Huerta-de-Soto

Pérez-Díaz, V., Álvarez-Miranda, B. y Chuliá, E. (1997). La opinión pública ante el sistema de pensiones. Barcelona: Servicio de Estudios La Caixa. Disponi- ble en: http://www.caixabankresearch. com/documents/10180/54279/ee10_ esp.pdf

Piñera, J. y Weinstein, A. (1996). Una propuesta de reformas del sistema de pensiones en España. Madrid: Círculo de Empresarios.

Preston, S. (1984). Children and the Elderly: divergent Paths for America's Dependents. Demography, 21, pp. 435-57. https://doi.org/10.2307/2060909

Royal Society (1992). Risk: Analysis, Perception and Management. Report of a Royal Society Study Group, London: Royal Society Publishing.

Sepúlveda Galeas, M. (2011). El riesgo como dispositivo de gobierno: neoprudencialismo y subjetivación. Revista de Psicología, 20, 2, pp. 103-124.

Sherden, W. (1998). The Fortune Sellers. The Big Business of Buying and Selling Predictions. New York: John Wiley \& Sons.

Van den Noord, P. y Herd R. (1993). Pension Liabilities in the Seven Major Economies. OECD Economics Department Working Papers, 142. París: OECD Publishing. https://doi.org/10.1787/083510523416

Van den Noord, P. y Herd R. (1994). Estimating Pension Liabilities: a Methodological Framework. OECD Economic Studies, 23, pp. 131-166. Disponible en: https://pdfs. semanticscholar.org/1347/84878315bcf 362e371ce4c92c0b26dd8be92.pdf

Vincent, J. (2003). OldAge. London: Routledge. https://doi.org/10.4324/9780203449929

Wynne, B. (1992). Misunderstood misunderstanding: social identities and public uptake of science. Public Understanding of Science, 1, pp. 281-304. https://doi. org/10.1088/0963-6625/1/3/004

Zubiri, I. y Civil, A. (2009). El sistema de pensiones español ante el reto del envejecimiento. Revista del Ministerio de Trabajo e Inmigración, 1, pp. 31-57.

Zubiri Oria, I. (2003). El futuro del sistema de pensiones en España. Madrid: Ministerio de Hacienda.

\section{Recursos de Internet}

BBC. Reith Lectures 1999. Runaway World, Lecture 2. Risk-Hong Kong. Professor Anthony Giddens. [En línea]. Disponible en: http://news.bbc.co.uk/hi/english/static/ events/reith_99/week2/week2.htm

Centro de Investigaciones Sociológicas. $\mathrm{Ba}$ rómetro de Marzo. Avance de resultados. Estudio no 2864. Marzo 2011. [En línea]. Disponible en: http://datos.cis. es/pdf/Es2864mar_A.pdf 